\title{
Homogeneous Hyperbolic Systems for Terahertz and Far-Infrared Frequencies
}

\author{
Leonid V. Alekseyev, ${ }^{1}$ Viktor A. Podolskiy, ${ }^{2,3}$ and Evgenii E. Narimanov ${ }^{1}$ \\ ${ }^{1}$ Electrical and Computer Engineering Department, Purdue University, West Lafayette, IN 47907, USA \\ ${ }^{2}$ Department of Physics, Oregon State University, Corvallis, OR 97331, USA \\ ${ }^{3}$ Department of Physics and Applied Physics, University of Massachusetts Lowell, Lowell, MA 01854, USA
}

Correspondence should be addressed to Evgenii E. Narimanov, evgenii@purdue.edu

Received 11 June 2012; Accepted 3 August 2012

Academic Editor: Pavel A. Belov

Copyright (C) 2012 Leonid V. Alekseyev et al. This is an open access article distributed under the Creative Commons Attribution License, which permits unrestricted use, distribution, and reproduction in any medium, provided the original work is properly cited.

We demonstrate that homogeneous naturally-occurring materials can form hyperbolic media, and can be used for nonmagnetic negative refractive index systems. We present specific realizations of the proposed approach for the THz and far-IR frequencies. The proposed structures operate away from resonance, thereby promising the capacity for low-loss devices.

Following the initial proposal by Veselago in 1968 [1], negative refraction materials spent over 30 years as a forlorn curiosity before being resurrected with renewed interest from both theoretical and experimental groups. Within the last decade it was realized that these materials (known also as left-handed materials), along with a broader classes of exotic media (known as epsilon near-zero materials, hyperbolic materials, etc.) possess unusual properties, some of which were not recognized at the time of their conceptions [2]. These properties include resonant enhancement of evanescent fields, strong suppression of diffraction, unusual modification to optical density of states, potentially enabling nearperfect imaging below the diffraction limit, and leading to a new class of optical devices [3], as well as nontrivial behavior in the nonlinear regime [4]. Despite initial controversy over the realizability of negative index materials (NIMs), successful proof of principle demonstrations have been accomplished [3, 5-8].

Existing designs for left-handed materials rely on achieving overlapping dipolar and magnetic resonances in subwavelength composites (metamaterials) [9, 10], or using photonic crystals near the bandgap $[3,11]$. Both of these approaches necessitate complicated $3 \mathrm{D}$ patterning of the medium with microstructured periodic arrays. Fabrication of such structures presents significant challenges even for
$\mathrm{GHz}$ applications, while manufacturing metamaterials for higher frequencies becomes harder still [12]. Furthermore, near-resonant operational losses impose severe limitations on the imaging resolution [13].

As an alternative to periodic systems, a waveguide-based implementation of a NIM was proposed [14], which obviates the need for negative magnetic permeability and does not require periodic patterning. This approach circumvents major manufacturing obstacles to achieving NIM behavior at terahertz or optical frequencies, and simultaneously opens a new avenue in imaging, sensing, and light emission applications $[15,16]$.

To achieve this behavior, the waveguide material must possess characteristics of a uniaxial medium with a significant anisotropy. Furthermore, this anisotropy must ensure that $\epsilon_{x}$ (the component of $\epsilon$ transverse to the planar waveguide) is negative, while $\epsilon_{\|} \equiv \epsilon_{y}\left(=\epsilon_{z}\right)$ (in-plane component) remains positive. TM modes in such waveguide undergo negative refraction in the waveguide plane, and propagate with negative phase velocity [14]. The materials with extremely strong anisotropy, hyperbolic materials, have since become a very active research area, enabling far-field imaging and focusing with subdiffraction resolution, broadband negative refraction, and superb control over light emission [17-22]. 
One of the key aspects in designing such a hyperbolic system is selecting the material for with strong underlying anisotropy. Several options have been proposed for the core material, in particular, nanostructured composites in a dielectric host and quantum well structures [14]. While being within the grasp of existing technology [23-25], the fabrication of such systems remains highly challenging.

In this work we present an alternative approach to hyperbolic media for $\mathrm{THz}$ and far-infrared domains based on naturally occurring materials with large dielectric anisotropy. In particular, we discuss the possibility of negative refraction in a system at approximately $20 \mu \mathrm{m}, 58 \mu \mathrm{m}$, and $255 \mu \mathrm{m}$ using, respectively, sapphire, bismuth, or triglycine sulfate in the waveguide core. Similar results were recently demonstrated at UV frequencies in graphite [26]. We focus on monocrystalline bismuth as an attractive option for manufacturing the NIM waveguide core thanks to its large anisotropy and availability of samples with high purity. We illustrate the perspectives of natural hyperbolic metamaterials on example of negative refraction in planar waveguide geometry.

In a planar waveguide with anisotropic dielectric core the wave vector components $k_{z}$ and $k_{y}$ are governed by the dispersion relation

$$
k_{z}^{2}+k_{y}^{2}=\epsilon \nu \frac{\omega^{2}}{c^{2}}
$$

with $\nu=\left(1-\kappa^{2} c^{2} / \epsilon_{\|} \omega^{2}\right)$, where $\omega$ is the frequency of light, $\epsilon=\epsilon_{x}\left(\epsilon_{\|}\right)$for the TM(TE) modes, $\kappa$ is the transverse mode parameter, and $k_{z}$ and $k_{y}$ lie in the waveguide plane. For perfectly conducting waveguide walls (a good approximation for silver and other metals at $\mathrm{THz}$ and far-IR frequencies [27]), $\kappa=m \pi / d$, where $m$ is an integer and $d$ is the thickness of the waveguide [14]. The effective refractive index for propagating waveguide modes in this system is given by $n_{\text {eff }}^{2}=\epsilon \nu$. To support propagating modes, $\epsilon$ and $\nu$ must have the same sign. The case $\epsilon>0, \nu>0$ is typically realized in an isotropic planar waveguide operating above cut-off. However, in the case $\epsilon<0, \nu<0$ negative refraction occurs [14], with refractive index given by

$$
n_{\text {eff }}=-\sqrt{\epsilon_{x} \nu} .
$$

Note that if $\kappa$ is regarded as the transverse wave vector component, (1) can be rewritten as a hyperbolic dispersion relation $k_{\perp}^{2} / \epsilon_{\|}-k_{\|}^{2} /\left|\epsilon_{x}\right|=\omega^{2} / c^{2}$, identified in [28] with the onset of negative refraction.

As follows from the definition of $\nu$, the propagation with negative phase velocity occurs only for TM modes when $\epsilon_{\|}>$ $0, \epsilon_{x}<0$. This behavior is observed in a number of materials where structural anisotropy strongly affects the dielectric response.

One example of such materials is triglycine sulfate (TGS), a compound widely used in fabricating infrared photodetectors. Spectroscopic studies of the crystal at low temperature have shown that phonon modes polarized parallel to the crystal's monoclinic $C_{2}$-axis significantly differ in frequency from phonons transverse to the axis. This results in a large anisotropy in the dielectric tensor along these directions. In particular, dielectric response for the field polarized along the $C_{2}$-axis features a resonance at $268 \mu \mathrm{m}$, which is absent if the incident field is polarized transverse to the $C_{2}$-axis [29]. Dielectric function $\epsilon_{x}$ in the vicinity of this resonance can be fitted with the Lorentz-Drude model [30], while $\epsilon_{x}$ in this region can be taken approximately constant $[29,31]$. Lorentz-Drude model parameters from [30] were used to construct Figure 1(a). As is evident from the figure, $\epsilon_{x}<0$, while $\epsilon_{\|}>0$ in the region $250 \leq \lambda \leq 268 \mu \mathrm{m}$. Furthermore, the imaginary part of $\epsilon$ becomes small away from the resonance, minimizing absorption. A TGS-filled waveguide with $C_{2}$-axis oriented perpendicular to the waveguide plane would support negative index propagation, while suffering from minimal propagation losses $\left(\operatorname{Im}[\epsilon] \sim 10^{-3}\right.$ at $\left.250 \mu \mathrm{m}\right)$.

Whereas the phonon anisotropy of TGS exists in the low- $\mathrm{THz}$ domain, for other materials, it may occur in a different spectral band. In particular, the strong anisotropy of the dielectric response of sapphire $\left(\mathrm{Al}_{2} \mathrm{O}_{3}\right)$ is also due to excitation of different phonon modes (polarized either parallel or perpendicular to the $c$-axis of the rhombohedral structure), but occurs around $20 \mu \mathrm{m}$. Figure 1(b) shows experimentally determined [32] $\epsilon_{\|}$and $\epsilon_{x}$ as functions of frequency. As with TGS, a region of $\epsilon_{x}<0, \epsilon_{\|}>0$ is evident in the experimental data. This potentially enables a sapphire-based waveguide NIM (with the $c$-axis of sapphire core perpendicular to the waveguide plane). Note that the minimum of the material absorption occurs in the frequency range of interest.

Anisotropic phonon excitations are not the only mechanism that can lead to strong dielectric anisotropy. Bismuth, a Group V semimetal with rhombohedral lattice and trigonal symmetry, exhibits such anisotropy due to a substantial difference in its electron effective masses along different directions in the crystal.

In the frequency region of interest, the spectral dependence of the electric permittivity of Bismuth can be adequately described by the Drude model,

$$
\epsilon=\epsilon_{L}\left(1-\frac{\omega_{\mathrm{pl}}^{2}}{\omega^{2}+i \omega \tau^{-1}}\right),
$$

with $\epsilon_{L}$ the lattice permittivity, $\omega_{\mathrm{pl}}=N e^{2} / \epsilon_{L} m_{\mathrm{eff}}$ the plasma frequency, and $\tau$ the relaxation time. These parameters are known from interferometric and reflectance studies of $\mathrm{Bi}$ samples. In particular, plasma frequency of pure Bismuth at $4 \mathrm{~K}$ was measured to be $158 \mathrm{~cm}^{-1}$ for the incident $E$-field polarized perpendicular to the trigonal axis, and $186 \mathrm{~cm}^{-1}$ for the field polarized parallel to the axis [33]. These values are in agreement with other experiments $[34,35]$. The lattice dielectric constant $\epsilon_{L}$ for the field perpendicular to the trigonal axis was found to be $110 \pm 10$ [35], in reasonable agreement with [34]. For polarization parallel to the trigonal axis, $\epsilon_{L}=76$ [36].

There can be substantial variation in the relaxation time $\tau$ depending on the purity of the sample. We take $\tau=$ $0.1 \mathrm{~ns}$ [35], however, this is a conservative estimate; for low temperatures, relaxation times over an order of magnitude greater have been reported as far back as 1975 [36]. Even with $\tau=0.1 \mathrm{~ns}$, the typical ratio of imaginary and real parts of the dielectric function in $\mathrm{Bi}$ is on the order of $0.1 \%$ in 

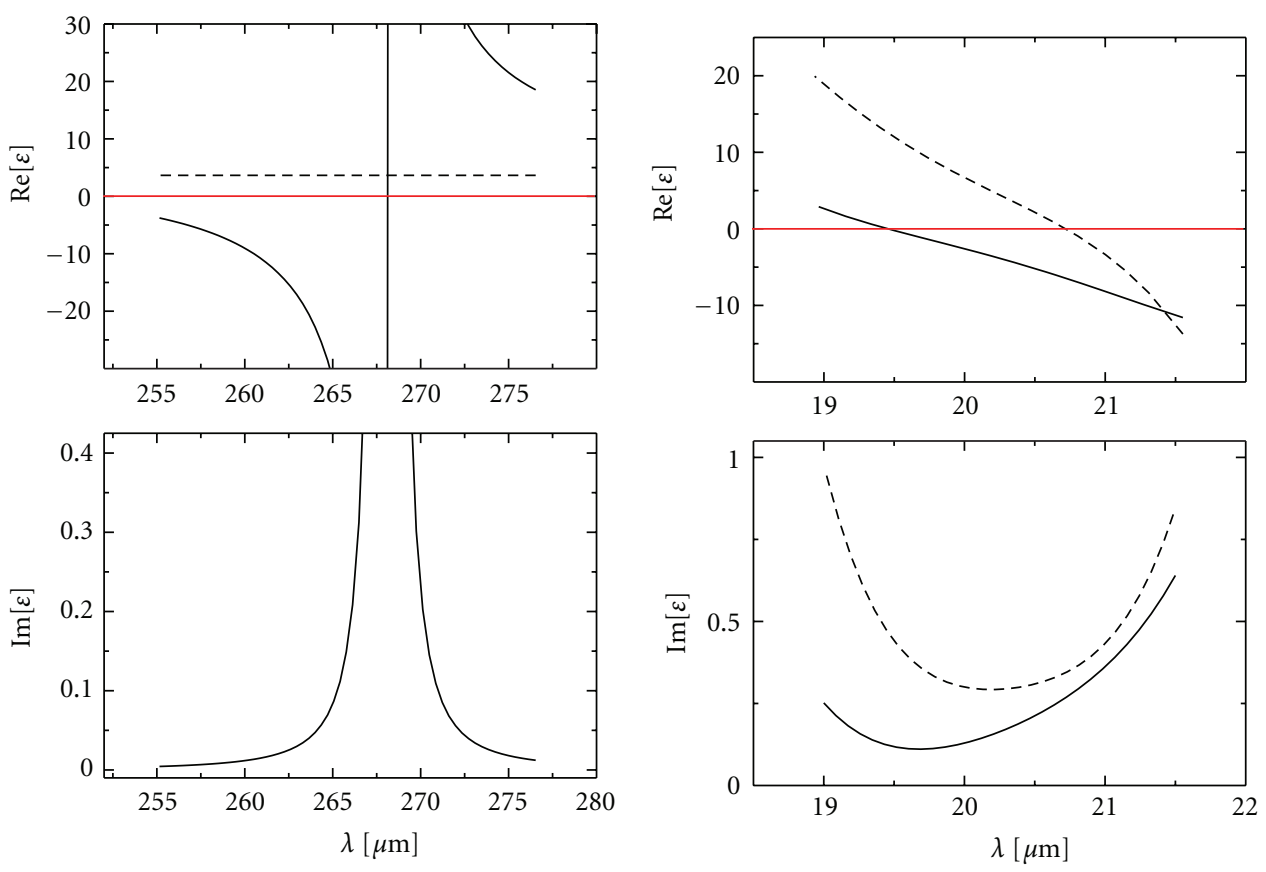

$---\varepsilon_{\|}$

$---\varepsilon_{\|}$

$\varepsilon_{\perp}$

(a)

(b)
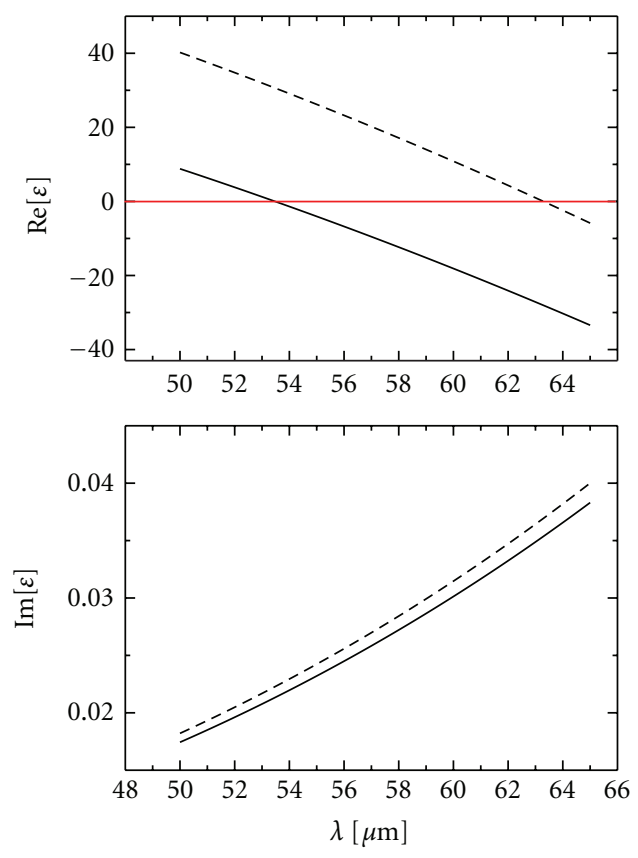

$---\varepsilon_{\|}$

$-\varepsilon_{\perp}$

(c)

Figure 1: (a) The real (top panel) and the imaginary (bottom panel) parts of the dielectric function of TGS; the monoclinic $C_{2}$-axis is along the "perpendicular" $(x)$ direction. (b) Same for sapphire; the crystallographic $c$-axis is along the "perpendicular" $(x)$ direction. (c) Same for monocrystalline Bismuth. 


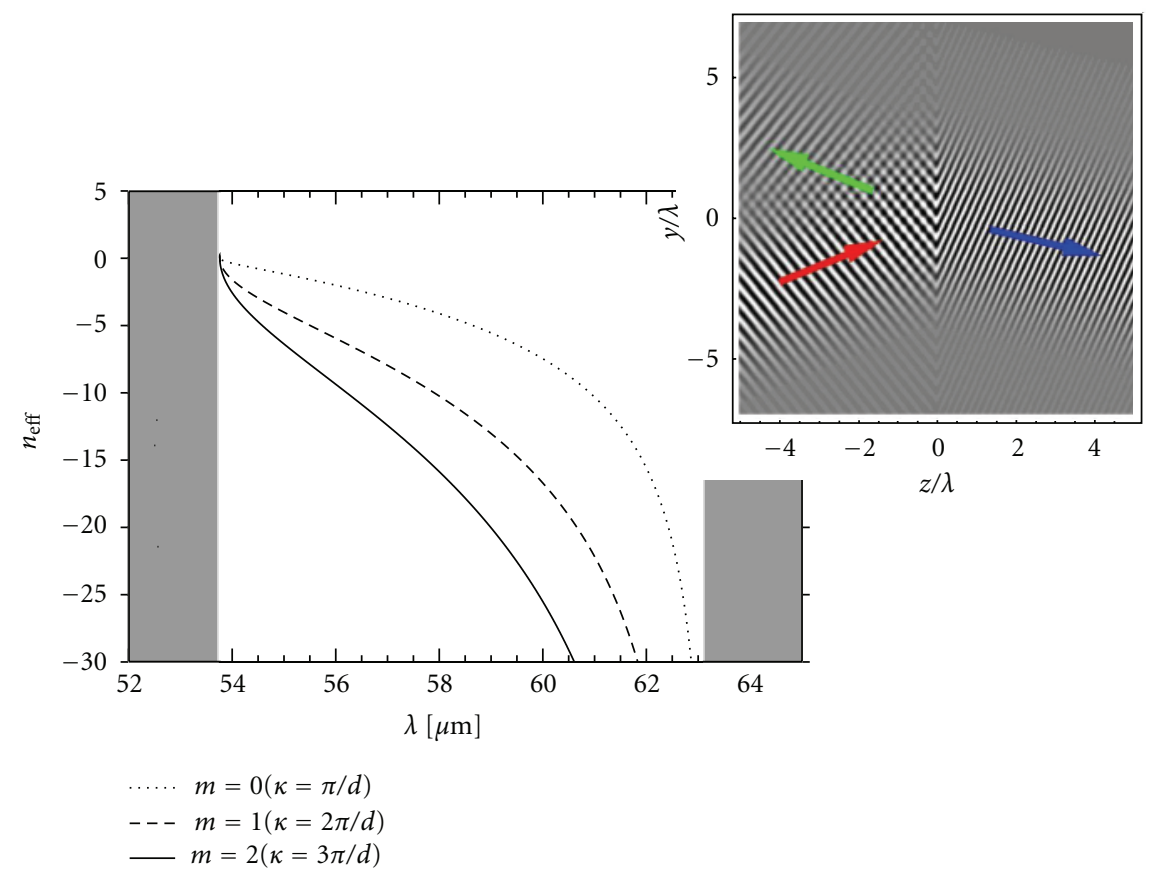

FIGURE 2: The effective refractive index of the three lowest order modes in a Bismuth waveguide. Inset: numerical simulation showing the refraction of a beam within a metallic waveguide (of thickness $d=4.5 \mu \mathrm{m}$ ) at an interface between an isotropic dielectric with $\epsilon=55$ and monocrystalline Bismuth; $\lambda=61 \mu \mathrm{m}$.

the frequency interval of interest, which enables many imaging and transmission applications [13]. It should also be noted that high-quality single-crystal films as thin as $1 \mu \mathrm{m}$, with the trigonal axis $\left(C_{3}\right)$ oriented perpendicular to the film plane, have been reported [37], thereby essentially solving the technological issues in fabricating the proposed negative index device.

Figure 1(c) shows the behavior of real and imaginary components of $\epsilon$ for Bi based on (3). The most prominent feature of these plots, the transition from $\epsilon>0$ to $\epsilon<0$, is determined by the highly anisotropic plasma frequency. This anisotropy creates a window between $\lambda=53.7 \mu \mathrm{m}$ and $63.2 \mu \mathrm{m}$ where $\epsilon<0$ for the $E$-field along the $C_{3}$-axis, while $\epsilon>0$ for $E$ transverse to $C_{3}$. The existence of such $10 \mu \mathrm{m}$ window was confirmed by direct measurement [38].

To allow for left-handed propagation in this frequency interval, $\mathrm{Bi}$ should be integrated into the core of a planar waveguide, with the $C_{3}$-axis oriented in the transverse direction. In Figure 2 we examine the behavior of the effective refractive index $n_{\text {eff }}(2)$ for the proposed subcritical $(d<\lambda / 2)$ waveguide structure with Bismuth core. Note that negative effective index is possible for all modes over the entire $\left(\epsilon_{x}<0, \epsilon_{\|}>0\right)$ range. Negative refraction behavior of our system was further confirmed by a numerical calculation of the electric field incident on the Bi waveguide. The results of this calculation are presented in Figure 2 (inset). We assume a TM wave with a Gaussian profile, mode-matched into the $\mathrm{Bi}$ waveguide in the transverse direction by, for example, propagating the beam from a metallic waveguide of the same thickness, filled with a regular dielectric. One can clearly see the negative refraction at the boundary. Furthermore, it is evident that attenuation of the transmitted wave is weak, as expected from low values of the imaginary part of the dielectric constant (Figure 1(c)).

In addition, this calculation shows that the NIM waveguide remains transparent despite the fact that transverse dimension of the waveguide is much smaller than the wavelength, which indicates strong confinement of the field within the core (since the cladding is assumed to be perfectly conducting). This behavior is not found in a subwavelength dielectric waveguide, where much of the field spreads into the cladding, or a subwavelength metallic waveguide, which does not support propagating modes. Such strong field confinement may find applications in photonic structures and nonlinear optics [39].

In conclusion, we have proposed a novel negative refraction system for several wavelengths from low-THz to far-IR. Our approach is nonmagnetic, avoids the use of periodic patterning, utilizes naturally occurring materials, and promises the capacity for low-loss devices.

\section{Acknowledgment}

This work was partially supported by ARO-Multidisciplinary University Research Initiative Grant nos. 50342-PH-MUR and W911NF-09-1-0539, NSF Grant nos. DMR-0134736, ECS-0400615, and ECCS-1102183, the Princeton Institute for the Science and Technology of Materials (PRISM), GRFOSU, and ACS-PRF. 


\section{References}

[1] V. G. Veselago, "The electrodynamics of substances with simultaneously negative values of $\varepsilon$ and $\mu$," Soviet Physics Uspekhi, vol. 10, no. 4, article 509, 1968.

[2] M. Noginov and V. A. Podolskiy, Eds., Tutorials in Metamaterials, Taylor \& Francis, Boca Raton, Fla, USA, 2012.

[3] P. V. Parimi, W. T. Lu, P. Vodo, and S. Sridhar, "Imaging by flat lens using negative refraction," Nature, vol. 426, no. 6965, article 404, 2003.

[4] I. R. Gabitov, R. A. Indik, N. M. Litchinitser, A. I. Maimistov, V. M. Shalaev, and J. E. Soneson, "Double-resonant optical materials with embedded metal nanostructures," Journal of the Optical Society of America B, vol. 23, no. 3, pp. 535-542, 2006.

[5] R. A. Shelby, D. R. Smith, and S. Schultz, "Experimental verification of a negative index of refraction," Science, vol. 292, no. 5514, pp. 77-79, 2001.

[6] V. M. Shalaev, W. Cai, U. K. Chettiar et al., "Negative index of refraction in optical metamaterials," Optics Letters, vol. 30, no. 24, pp. 3356-3358, 2005.

[7] E. Schonbrun, T. Yamashita, W. Park, and C. J. Summerss, "Negative-index imaging by an index-matched photonic crystal slab," Physical Review B, vol. 73, no. 19, Article ID 195117, 6 pages, 2006.

[8] G. Dolling, C. Enkrich, M. Wegener, C. M. Soukoulis, and S. Linden, "Simultaneous negative phase and group velocity of light in a metamaterial," Science, vol. 312 , no. 5775 , pp. $892-$ $894,2006$.

[9] D. R. Smith, W. J. Padilla, D. C. Vier, S. C. Nemat-Nasser, and S. Shultz, "Composite medium with simultaneously negative permeability and permittivity," Physical Review Letters, vol. 84, no. 18, pp. 4184-4187, 2000.

[10] C. G. Parazzoli, R. B. Greegor, K. Li, B. E. C. Koltenbah, and M. Tanielian, "Experimental verification and simulation of negative index of refraction using Snell's law," Physical Review Letters, vol. 90, no. 10, Article ID 107401, 4 pages, 2003.

[11] M. Notomi, "Theory of light propagation in strongly modulated photonic crystals: refractionlike behavior in the vicinity of the photonic band gap," Physical Review B, vol. 62, no. 16, pp. 10696-10705, 2000.

[12] A. Boltasseva, "Fabrication of optical metamaterials," in Tutorials in Metamaterials, M. Noginov and V. A. Podolskiy, Eds., Taylor \& Francis, Boca Raton, Fla, USA, 2012.

[13] V. A. Podolskiy and E. E. Narimanov, "Near-sighted superlens," Optics Letters, vol. 30, no. 1, pp. 75-77, 2005.

[14] V. A. Podolskiy and E. E. Narimanov, "Strongly anisotropic waveguide as a nonmagnetic left-handed system ," Physical Review B, vol. 71, no. 20, Article ID 201101(R), 4 pages, 2005.

[15] V. A. Podolskiy, "Hyperbolic metamaterials," in Tutorials in Metamaterials, M. Noginov and V. A. Podolskiy, Eds., Taylor \& Francis, Boca Raton, Fla, USA, 2012.

[16] L. V. Alekseyev and E. E. Narimanov, "Radiative decay engineering in metamaterials," in Tutorials in Metamaterials, M. Noginov and V. A. Podolskiy, Eds., Taylor \& Francis, Boca Raton, Fla, USA, 2012.

[17] Z. Jacob, L. V. Alekseyev, and E. Narimanov, "Optical hyperlens: far-field imaging beyond the diffraction limit," Optics Express, vol. 14, no. 18, pp. 8247-8256, 2006.

[18] S. Thongrattanasiri and V. A. Podolskiy, "Hypergratings: nanophotonics in planar anisotropic metamaterials," Optics Letters, vol. 34, no. 7, pp. 890-892, 2009.
[19] A. Salandrino and N. Engheta, "Far-field subdiffraction optical microscopy using metamaterial crystals: theory and simulations," Physical Review B, vol. 74, no. 7, Article ID 075103, 2006.

[20] Z. Liu, H. Lee, Y. Xiong, C. Sun, and X. Zhang, "Far-field optical hyperlens magnifying sub-diffraction-limited objects," Science, vol. 315, no. 5819, article 1686, 2007.

[21] H. N. S. Krishnamoorthy, Z. Jacob, E. Narimanov, I. Kretzschmar, and V. M. Menon, "Topological transitions in metamaterials," Science, vol. 336, no. 6078, pp. 205-209, 2012.

[22] E. E. Narimanov, H. Li, Y. A. Barnakov, and M. A. Noginov, "Darker than black: radiation-absorbing metamaterial," in Proceedings of the Lasers and Electro-Optics/Quantum Electronics and Laser Science Conference: 2010 Laser Science to Photonic Applications (CLEO/QELS'10), Optical Society of America, San Jose, Calif, USA, May 2010, presentation \#QPDA6.

[23] P. Evans, W. R. Hendren, R. Atkinson et al., "Growth and properties of gold and nickel nanorods in thin film alumina," Nanotechnology, vol. 17, no. 23, pp. 5746-5753, 2006.

[24] J. Yao, Z. Liu, Y. Liu et al., "Optical negative refraction in bulk metamaterials of nanowires," Science, vol. 321, no. 5891, p. 930, 2008.

[25] A. J. Hoffman, L. Alekseyev, S. S. Howard et al., "Negative refraction in semiconductor metamaterials," Nature Materials, vol. 6, no. 12, pp. 946-950, 2007.

[26] J. Sun, J. Zhou, B. Li, and F. Kang, "Indefinite permittivity and negative refraction in natural material: graphite," Applied Physics Letters, vol. 98, no. 10, Article ID 101901, 3 pages, 2011.

[27] R. Wangberg, J. Elser, E. E. Narimanov, and V. A. Podolskiy, "Nonmagnetic nanocomposites for optical and infrared negative-refractive-index media," Journal of the Optical Society of America B, vol. 23, no. 3, pp. 498-505, 2006.

[28] C. Luo, S. G. Johnson, and J. D. Joannopoulos, "All-angle negative refraction without negative effective index," Physical Review B, vol. 65, no. 20, Article ID 201104(R), 4 pages, 2002.

[29] A. Hadni and X. Gerbaux, "Far IR excitation of longitudinal optical phonons in triglycine sulphate," Ferroelectrics, vol. 248, no. 1, pp. 15-26, 2000.

[30] X. Gerbaux, M. Tazawa, and A. Hadni, "Far IR transmission measurements on triglycine sulphate (TGS), at 5K," Ferroelectrics, vol. 215, no. 1, pp. 47-63, 1998.

[31] T. Dumelow, J. A. P. da Costa, and V. N. Freire, "Slab lenses from simple anisotropic media," Physical Review B, vol. 72, no. 23, Article ID 235115, 8 pages, 2005.

[32] M. Schubert, T. E. Tiwald, and C. M. Herzinger, "Infrared dielectric anisotropy and phonon modes of sapphire," Physical Review B, vol. 61, no. 12, pp. 8187-8201, 2000.

[33] W. S. Boyle, A. D. Brailsford, and J. K. Galt, "Dielectric anomalies and cyclotron absorption in the infrared: observations on bismuth," Physics Reviews, vol. 109, no. 4, pp. 1396-1398, 1958.

[34] W. S. Boyle and A. D. Brailsford, "Far infrared studies of bismuth,” Physics Reviews, vol. 120, no. 6, pp. 1943-1949, 1960.

[35] V. D. Kulakovskii and V. D. Egorov, "Plasma reflection in bismuth and bismuth-antimony alloys," Soviet Physics—Solid State, vol. 15, no. 7, p. 1368, 1974.

[36] V. S. Edelman, "Electrons in bismuth," Advances in Physics, vol. 25, no. 6, pp. 555-613, 1976.

[37] F. Y. Yang, K. Liu, K. Hong, D. H. Reich, P. C. Searson, and C. L. Chien, "Large magnetoresistance of electrodeposited singlecrystal bismuth thin films," Science, vol. 284, no. 5418, pp. 1335-1337, 1999. 
[38] E. Gerlach, P. Grosse, M. Rautenberg, and W. Senske, "Dynamical conductivity and plasmon excitation in Bi," Physica Status Solidi (b), vol. 75, no. 2, pp. 553-558, 1976.

[39] A. A. Govyadinov and V. A. Podolskiy, "Metamaterial photonic funnels for subdiffraction light compression and propagation," Physical Review B, vol. 73, no. 15, Article ID 155108, 5 pages, 2006. 

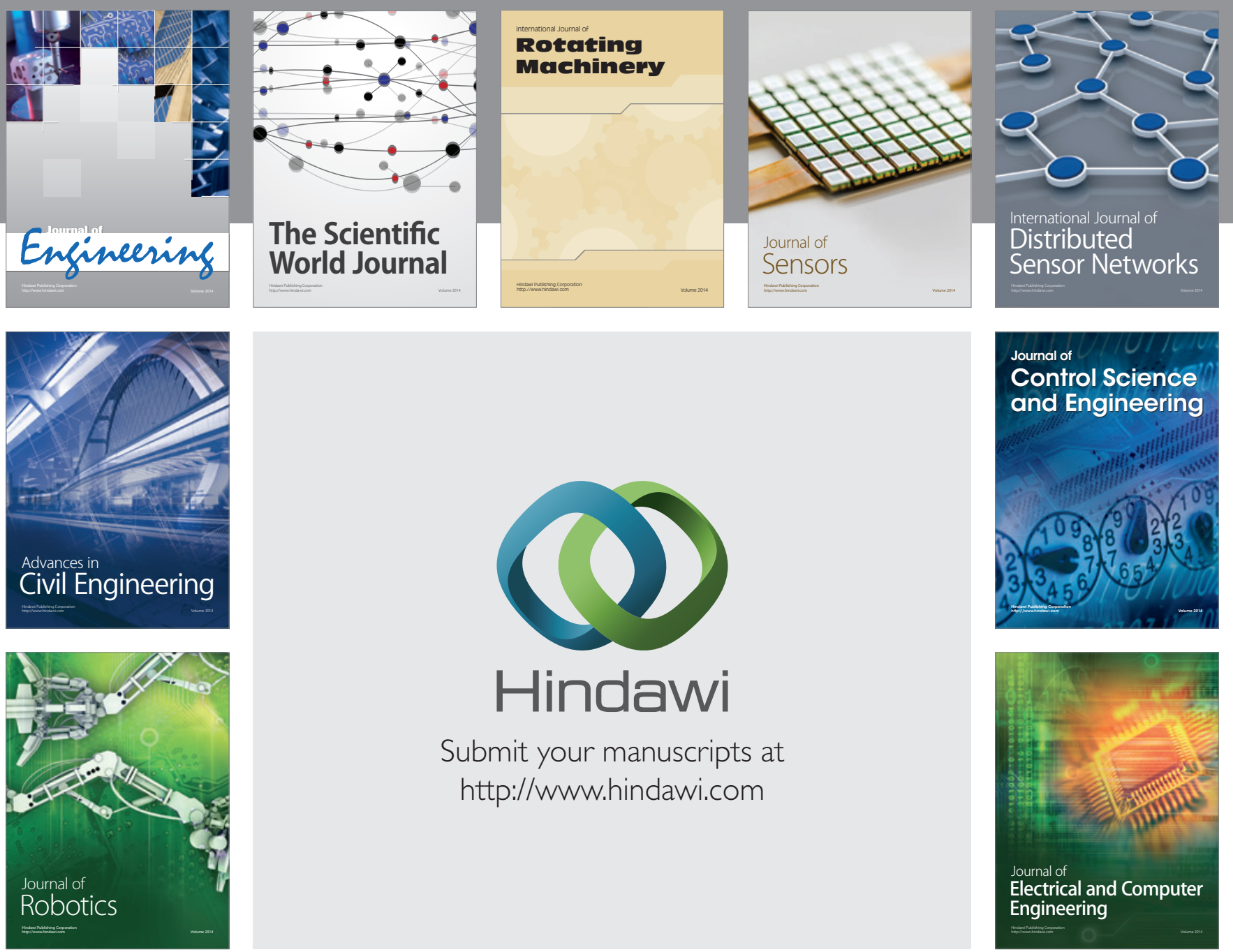

Submit your manuscripts at

http://www.hindawi.com
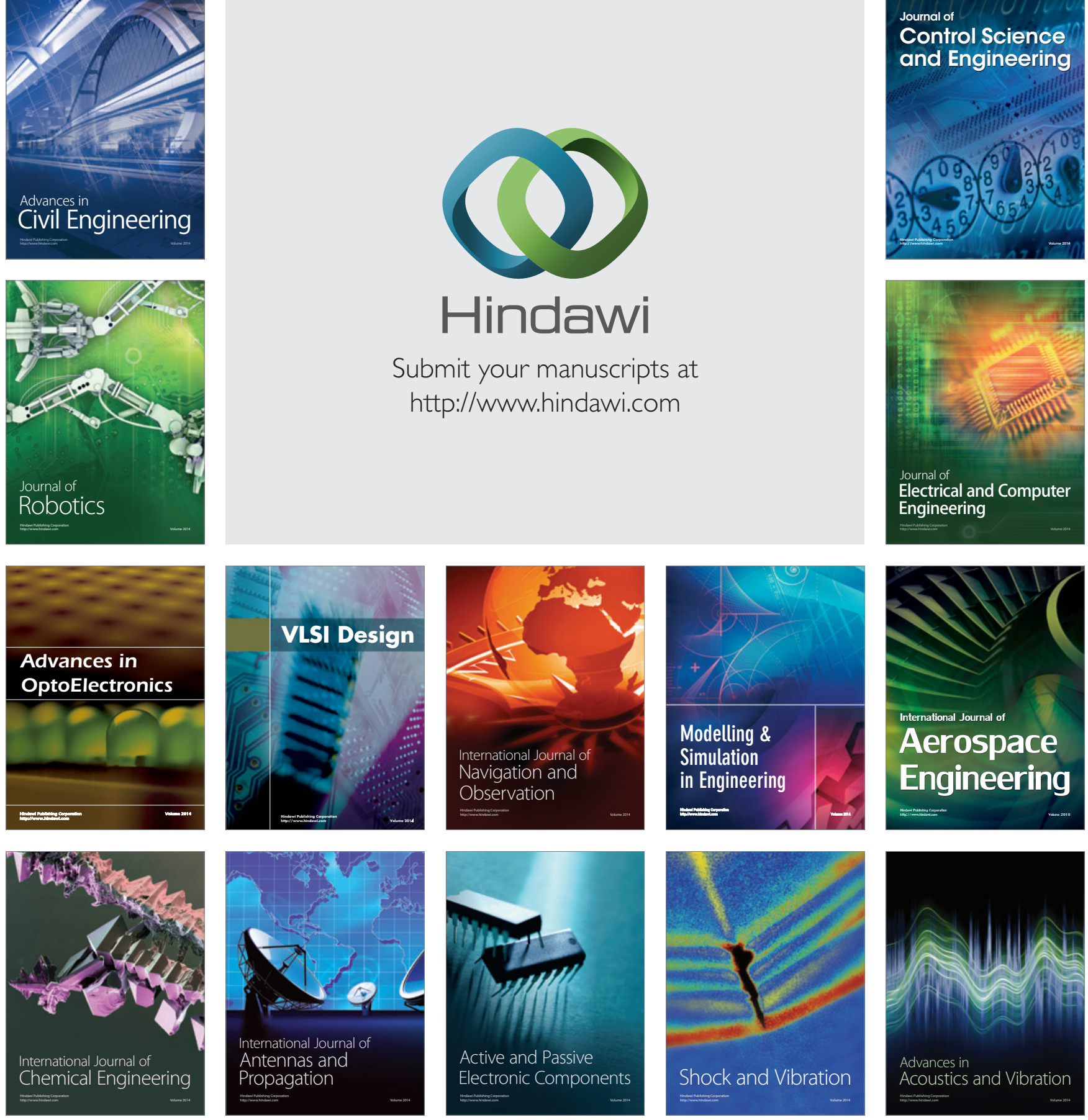